\title{
COMBINATORIAL ORTHOGONAL EXPANSIONS
}

\author{
A. DE MÉDICIS AND D. STANTON \\ (Communicated by Jeffry N. Kahn)
}

\begin{abstract}
The linearization coefficients for a set of orthogonal polynomials are given explicitly as a weighted sum of combinatorial objects. Positivity theorems of Askey and Szwarc are corollaries of these expansions.
\end{abstract}

\section{INTRODUCTION}

Given a set of orthogonal polynomials $p_{n}(x)$, the linearization coefficients $a_{m n}^{k}$ are given by

$$
p_{m}(x) p_{n}(x)=\sum_{k} a_{m n}^{k} p_{k}(x)
$$

Askey [1] and Szwarc $[4,5]$ have given sufficient conditions on the three-term recurrence relation coefficients $\alpha_{n}, \beta_{n}$, and $\gamma_{n}$ in

$$
\alpha_{n+1} p_{n+1}(x)=\left(x-\beta_{n}\right) p_{n}(x)-\gamma_{n-1} p_{n-1}(x)
$$

so that $a_{m n}^{k}$ is non-negative. In this paper we give in Theorem 1 and Theorem 2 explicit formulas for $a_{m n}^{k}$ as a polynomial in the $\alpha_{j}$ 's, $\beta_{j}$ 's and $\gamma_{j}$ 's, which give these theorems.

The idea is to represent $a_{m n}^{k}$ as a generating function of paths, whose weights are products of differences. Monotonicity hypotheses on the coefficients force the weights to be individually positive, these are the conditions in [1] and [4]. For example, if $p_{n}(x)$ is monic; $\alpha_{n}=1, \beta_{n}=b_{n}$, and $\gamma_{n}=\lambda_{n+1}$, we have

$$
\begin{aligned}
a_{33}^{3}= & \left(b_{3}-b_{0}\right)\left(b_{3}-b_{1}\right)\left(b_{3}-b_{2}\right)+\left(b_{3}-b_{0}\right) \lambda_{4}+\left(b_{3}-b_{0}\right)\left(\lambda_{3}-\lambda_{2}\right) \\
& +\left(b_{4}-b_{1}\right) \lambda_{4}+\left(b_{3}-b_{2}\right) \lambda_{4}+\left(b_{2}-b_{1}\right) \lambda_{3}+\left(b_{3}-b_{2}\right)\left(\lambda_{3}-\lambda_{1}\right) .
\end{aligned}
$$

If $b_{j}$ and $\lambda_{j}>0$ are increasing, then $a_{33}^{3}$ is non-negative, see [1].

Received by the editors August 19, 1994.

1991 Mathematics Subject Classification. Primary 42C05, 05E35.

The first author's work was supported by NSERC funds.

The second author's work was supported by NSF grant DMS-9001195. 


\section{THE THEOREMS}

We first recall some terminology and results in [3] and [6].

We let $L$ denote the positive definite linear functional on the space of polynomials which corresponds to the orthogonal polynomials (1.1). So $L\left(x^{n}\right)=\mu_{n}$, the $n t h$ moment of a measure for $p_{n}(x)$. It is easy to see that

$$
a_{m n}^{k}=L\left(p_{m} p_{n} p_{k}\right) / L\left(p_{k} p_{k}\right) .
$$

Since $L\left(p_{k} p_{k}\right)=\gamma_{0} \cdots \gamma_{k-1} / \alpha_{1} \cdots \alpha_{k}>0$, we find instead $L\left(p_{m} p_{n} p_{k}\right)$.

Viennot [6] gave a combinatorial interpretation for the polynomials $p_{n}(x)$ and their moments $\mu_{n}$, in terms of pavings and Motzkin paths respectively. We review these terms below.

A Motzkin path $P$ is a lattice path in the plane, which lies at or above the $x$-axis, and has steps of $(1,0)$ (horizontal $=H),(1,1)(\mathrm{up}=U)$, or $(1,-1)($ down $=D)$. The weight of a path $P, w(P)$, is defined by the product of the weights of its individual edges,

$$
w(P)=\prod_{\text {edges } e} w(e) .
$$

A paving $\pi$ of the integers $\{1, \cdots, k\}$ is a collection of disjoint sets of cardinalities 1 (called monominos), and 2 (called dominos). The elements of a domino must be consecutive integers. For example, $\{\{2,3\},\{5\},\{6,7\},\{9\}\}$ is a paving of $\{1, \cdots, 9\}$. Points not in any of the sets are called isolated. The weight of a paving is defined to be the product of the individual weights of the monominos, dominos, and isolated points.

For Askey's theorem we need a special weight on edges $e$ of a Motzkin path. Suppose the path $P$ begins at $(0, m)$ and ends at $(k, n)$. We define

$$
w \text { (edge starting at }(i, j))=\left\{\begin{array}{l}
\left(b_{j}-b_{i}\right) \text { if the edge is } H, \\
\left(\lambda_{j}-\lambda_{i+1}\right) \text { if the edge is } D, \text { and followed by } U, \\
\lambda_{j} \text { if the edge is } D, \text { and not followed by } U, \\
1 \text { if the edge is } U .
\end{array}\right.
$$

Theorem 1. Suppose that $\alpha_{n}=1, \beta_{n}=b_{n}$, and $\gamma_{n}=\lambda_{n+1}$. Then

$$
L\left(p_{m} p_{n} p_{k}\right)=\lambda_{1} \cdots \lambda_{n} \sum_{P} w(P)
$$

where $P$ is a Motzkin path from $(0, m)$ to $(k, n)$, and $w(P)$ is given by $(2.1)$ and $(2.2)$.

For example, if $k=m=n=3$ in Theorem 1, there are seven Motzkin paths from $(0,3)$ to $(3,3)$ : $H H H, H U D, H D U, U H D, U D H, D H U, D U H$. The weights of these seven paths are the seven terms in (1.2).

Proof of Theorem 1. One can prove that both sides in Theorem 1 have the same recurrence relation, which is given in [1].

An alternative proof is to use Viennot's combinatorial interpretation for $L\left(p_{m} p_{n} p_{k}\right) / \lambda_{1} \cdots \lambda_{n}[6]$. It is the generating function for ordered pairs $(P, \pi)$, 
where $P$ is a Motzkin path from $(0, m)$ to $(l, n)$, and $\pi$ is a paving of the integers $\{1, \cdots, k\}$ with $l$ isolated integers. The weight of $(P, \pi)$ is the product of the weights of $P$ and $\pi$. In $P$, an up edge starting at $(i, j)$ has weight 1 , a down edge $\lambda_{j}$, and an across edge $b_{j}$. For $\pi$, a monomino at $\{i\}$ has weight $-b_{i-1}$, and a domino at $\{i, i+1\}$ has weight $-\lambda_{i}$.

Given $(P, \pi)$ we create a unique path $P^{\prime}$ by inserting in $P$, as the ith step of $P^{\prime}$, an $H$ edge if $\pi$ has a monomino in position $i$. If $\pi$ has a domino starting in position $i$, we insert two steps, $D U$, in $P$, for the $i t h$ and $(i+1) s t$ steps of $P^{\prime}$. The result is a single path $P^{\prime}$ from $(0, m)$ to $(k, n)$. The weight of the path is given by $(2.2)$ : the negative terms correspond to the weight in $\pi$, the positive terms to the weight in $P$.

It is easy to see that Theorem 1 implies Askey's theorem.

Corollary 1. If $\lambda_{j}$ and $b_{j}$ are increasing, with $\lambda_{j}>0$, then $a_{m n}^{k} \geq 0$.

Proof. We can assume by symmetry that $k \leq n$, Then it is clear that each vertex $(i, j)$ in $P$ satisfies $i \leq j$. Thus all weights are non-negative if the $b_{j}$ 's and $\lambda_{j}$ 's are increasing.

Theorem 1 can be restated in terms of walks of length $m$ on the non-negative integers, starting at $k$, and ending at $n$, with steps of size $+1,-1$, or 0 .

We let $p_{n}^{\prime}(x)$ be another set of orthogonal polynomials satisfying

$$
\alpha_{n+1}^{\prime} p_{n+1}^{\prime}(x)=\left(x-\beta_{n}^{\prime}\right) p_{n}^{\prime}(x)-\gamma_{n-1}^{\prime} p_{n-1}^{\prime}(x) .
$$

More generally, we consider

$$
p_{m}(x) p_{k}^{\prime}(x)=\sum_{n} b_{m k}^{n} p_{n}(x)
$$

It is clear that $b_{m k}^{n}=L\left(p_{m} p_{k}^{\prime} p_{n}\right) / L\left(p_{n} p_{n}\right)$. We will give an interpretation for $L\left(p_{m} p_{k}^{\prime} p_{n}\right)$, which is non-negative when $b_{m k}^{n}$ is, since $L$ is positive definite.

We generalize Szwarc's theorem by finding a combinatorial interpretation for $L\left(p_{m} p_{k}^{\prime} p_{n}\right)$ in (2.3). A generalized Motzkin path allows a fourth type of edge: $H H$ (across by two units). We define a weight $v(P)$ on generalized Motzkin paths from $(0, m)$ to $(k, n)$ again as a product of weights of edges,

$v($ edge starting at $(i, j))$

$$
=\left\{\begin{array}{l}
\left(\beta_{j}-\beta_{i}^{\prime}\right) \text { if the edge is } H, \\
\left(\gamma_{j}-\alpha_{i}^{\prime}\right) \text { if the edge is } U, \text { and preceded by } D, \\
\gamma_{j} \text { if the edge is } U, \text { and not preceded by } D, \\
\left(\alpha_{j}-\alpha_{i}^{\prime}\right) \text { if the edge is } D, \text { and preceded by } U, \\
\alpha_{j} \text { if the edge is } D, \text { and not preceded by } U, \\
\left(\alpha_{j}+\gamma_{j}-\alpha_{i}^{\prime}-\gamma_{i}^{\prime}\right) \alpha_{i+1}^{\prime} \text { if the edge is } H H, \text { preceded by } U \text { or } D, \\
\left(\alpha_{j}+\gamma_{j}-\gamma_{i}^{\prime}\right) \alpha_{i+1}^{\prime} \text { if the edge is } H H, \text { not preceded by } U \text { or } D .
\end{array}\right.
$$


Theorem 2. We have

$$
L\left(p_{m} p_{n} p_{k}^{\prime}\right)=\frac{\gamma_{0} \cdots \gamma_{k-1}}{\alpha_{1} \cdots \alpha_{m} \alpha_{1}^{\prime} \cdots \alpha_{k}^{\prime}} \sum_{P} v(P)
$$

where $P$ is a generalized Motzkin path from $(0, m)$ to $(k, n)$, and $v(P)$ is given by (2.1) and (2.4).

Proof. Again we will use Viennot's interpretation for

$$
L\left(p_{m} p_{n} p_{k}^{\prime}\right) \alpha_{1} \cdots \alpha_{m} / \gamma_{0} \cdots \gamma_{k-1}
$$

The weights on the edges, monominos, and dominos slightly change. Let $P^{\prime}$ denote the Motzkin path and $\pi^{\prime}$ the paving. In $P^{\prime}$, the $U, D$, and $H$ edges starting at $(i, j)$ have weights $\gamma_{j}, \alpha_{j}$, and $\beta_{j}$ respectively. In $\pi^{\prime}$, a monomino $\{i\}$ has weight $-\beta_{i-1}^{\prime} / \alpha_{i}^{\prime}$, a domino $\{i, i+1\}$ has weight $-\gamma_{i-1}^{\prime} \alpha_{i}^{\prime} /\left(\alpha_{i}^{\prime} \alpha_{i+1}^{\prime}\right)$, and an isolated point $i$ has weight $1 / \alpha_{i}^{\prime}$. Note that every paving has a factor of $1 / \alpha_{1}^{\prime} \cdots \alpha_{k}^{\prime}$. We therefore disregard the denominators of the weights of the pavings, and put this constant factor in the statement of Theorem 2 .

As in Theorem 1, we will merge pavings $\pi^{\prime}$ with the paths $P^{\prime}$ to create a generalized Motzkin path $P$ whose weights are given by (2.1) and (2.5):

$$
u(\text { edge starting at }(i, j))=\left\{\begin{array}{l}
\left(\beta_{j}-\beta_{i}^{\prime}\right) \text { if the edge is } H, \\
\gamma_{j} \text { if the edge is } U, \\
\alpha_{j} \text { if the edge is } D \\
-\gamma_{i}^{\prime} \alpha_{i+1}^{\prime} \text { if the edge is } H H
\end{array}\right.
$$

The basic idea is to insert certain edges into $P^{\prime}$ to create $P$, while simultaneously deleting all monominos and dominos in $\pi^{\prime}$. This is done by inserting an $H$ edge in $P^{\prime}$ starting at $(i, j)$, if $\pi^{\prime}$ has the monomino $\{i+1\}$. We insert an $H H$ edge in $P^{\prime}$ starting at $(i, j)$, if $\pi^{\prime}$ has the domino $\{i+1, i+2\}$. We obtain a multiset of generalized Motzkin paths $P:(0, m) \rightarrow(k, n)$, from which the multiplicities are eliminated by using the weight (2.5).

Let $S$ be the set of all generalized Motzkin paths from $(0, m)$ to $(k, n)$. We just found that the linearization coefficients are, up to a constant, the generating function for $S$ with weight (2.5). We want weight (2.4) instead of (2.5). We will do this via an involution.

The (2.4) weights of the edges of $P \in S$ are not monomials, instead they are sums of monomials. Thus we can consider the multiset $M_{1}$ of paths $P \in S$, where the multiplicity of $P$ in $M_{1}$ is the product of the number of monomials in the weight of the edges $e \neq H$ of $P$. The weight of any element of $M_{1}$ is the product of a choice of monomials for each edge. On $M_{1}$ we will construct a weight-preserving sign-reversing involution, whose fixed point set consists of all paths $P$ exactly once, with weights $(2.5)$.

It remains to give the involution $\Phi$ on the multiset $M_{1}$ of paths $P$. Note that we want to eliminate all weights in the edges that include $\alpha^{\prime}$, except for the $-\gamma_{i}^{\prime} \alpha_{i+1}^{\prime}$ term in $H H$. Scan the path $P$ from right to left, and find the first such term in the choice of monomials for the weights. Suppose the edge containing this term is $H H$, preceded by $U$ or $D$. From (2.5), the weight we need to eliminate is one term 
from $\left(\alpha_{j}+\gamma_{j}-\alpha_{i}^{\prime}\right) \alpha_{i+1}^{\prime}$. If the preceding edge is $D$, replacing the $H H$ edge by a pair $U D$ will cancel the $\left(\gamma_{j}-\alpha_{i}^{\prime}\right) \alpha_{i+1}^{\prime}$ terms, while replacing the $H H$ edge by $D U$ will cancel the $\alpha_{j} \alpha_{i+1}^{\prime}$ term. Similarly, if the preceding edge to $H H$ is $U$, replacing $H H$ by $U D$ and $D U$ will cancel the $\gamma_{j} \alpha_{i+1}^{\prime}$ and $\left(\alpha_{j}-\alpha_{i}^{\prime}\right) \alpha_{i+1}^{\prime}$ terms, respectively. If the first edge containing $\alpha^{\prime}$ is $H H$, not preceded by $U$ or $D$, we must eliminate $\left(\alpha_{j}+\gamma_{j}\right) \alpha_{i+1}^{\prime}$. This time replacing $H H$ by $D U$ and $U D$ eliminates a single term each.

This defines $\Phi(P)=Q$, when the first appropriate $\alpha^{\prime}$ edge of $P$ is $H H$. If the first appropriate $\alpha^{\prime}$ edge of $P$ is not $H H$, then $\alpha^{\prime}$ must be a choice of weight from a $D U$ or $U D$. Then we invert the previous case. It is easy to check that the involution $\Phi$ is well defined on $M_{1}$, with the stated fixed points.

Corollary 2 generalizes [4, Theorem 2].

Corollary 2. If $\alpha_{i}, \alpha_{i}^{\prime}, \gamma_{i}, \gamma_{i}^{\prime}>0, \beta_{j} \geq \beta_{i}^{\prime}, \alpha_{j} \geq \alpha_{i}^{\prime}, \alpha_{j}+\gamma_{j} \geq \alpha_{i}^{\prime}+\gamma_{i}^{\prime}, \gamma_{j} \geq \alpha_{i}^{\prime}$, for $j \geq i$, and $k \leq \max \{m, n\}$, then $b_{m k}^{n} \geq 0$.

Proof. Assume $k \leq n$. The inequalities insure that the individual weights in Theorem 2 are positive, since the indices of the primed variables cannot be greater than the indices of the unprimed variables. By symmetry we obtain the $k \leq \max \{m, n\}$ case.

The connection coefficient problem is the $m=0$ special case of Theorem 2 . Nonzero coefficients occur only for $k \geq n$. In this case, along our path $P$, vertices $(i, j)$ satisfy $i \geq j$, so we assume the inequalities of Corollary 2 hold in this range. This implies Askey's theorem in [2].

The theorems in [5] can also be generalized, for example:

Corollary 3. If $\beta_{j}=\beta_{i}^{\prime}=0, \alpha_{i}, \alpha_{i}^{\prime}, \gamma_{i}, \gamma_{i}^{\prime}>0, \alpha_{2 j} \geq \alpha_{2 i}^{\prime}, \alpha_{2 j+1} \geq \alpha_{2 i+1}^{\prime}, \alpha_{2 j}+$ $\gamma_{2 j} \geq \alpha_{2 i}^{\prime}+\gamma_{2 i}^{\prime}, \alpha_{2 j+1}+\gamma_{2 j+1} \geq \alpha_{2 i+1}^{\prime}+\gamma_{2 i+1}^{\prime}, \gamma_{2 j} \geq \alpha_{2 i}^{\prime}, \gamma_{2 j+1} \geq \alpha_{2 i+1}^{\prime}$, for $j \geq i$, $m$ is even, and $k \leq n$, then $b_{m k}^{n} \geq 0$.

Proof. Under the assumption that $m$ is even, and all $\beta^{\prime} s=0$, all vertices $(i, j)$ on the path $P$ of Theorem 2 have the property that $i$ and $j$ have the same parity.

\section{REFERENCES}

1. R. Askey, Linearization of the product of orthogonal polynomials, Problems in Analysis ( $\mathrm{R}$ Gunning, ed.), Princeton Univ. Press, Princeton, NJ, 1970, pp. 223-228. MR 49:9525

2. Orthogonal expansions with positive coefficients. II, SIAM J. Math. Anal. 2 (1971), 340-346. MR 45:5650

3. T. Chihara, An introduction to orthogonal polynomials, Gordon and Breach, New York, 1978. MR 58:1979

4. R. Szwarc, Orthogonal polynomials and a discrete boundary value problem I, SIAM J. Math. Anal. 23 (1992), 959-964. MR 93i:33007

5. Urthogonal expansions and a discrete boundary value problem II, SIAM J. Math. Anal. 23 (1992), 965-969. MR 93i:33007

6. G. Viennot, Une théorie combinatoire des polynômes orthogonaux généraux, Lecture Notes, UQAM, 1983.

School of Mathematics, University of Minnesota, Minneapolis, Minnesota 55455

Current address, Anne de Médicis: LACIM, Département de Mathématiques, UQAM, C.P.

8888, succ. A, Montréal, Québec, Canada H3C 3P8

E-mail address: medicis@lacim.uqam.ca

E-mail address: stanton@s2.math.umn.edu 